\title{
Flux Tube Solutions in Kaluza-Klein Theory
}

\author{
V. Dzhunushaliev * \\ Dept. of Phys. VCU, Richmond, VA 23284-2000, USA \\ and \\ Theor. Phys. Dept. KSNU, 720024, Bishkek, Kyrgyzstan \\ D. Singleton ${ }^{\dagger}$ \\ Dept. of Phys. CSU Fresno, 2345 East San Ramon Ave., Fresno, CA 93740-8031 USA
}

(March 26, 2022)

\begin{abstract}
Spherically symmetric, vacuum solutions in 5D and 7D Kaluza-Klein theory are obtained. These solutions are flux tubes with constant cross-sectional size, located between $(+)$ and (-) Kaluza-Klein "electrical" and "magnetic" charges disposed respectively at $r= \pm \infty$ and filled with constant Kaluza-Klein "electrical" and "magnetic" fields. These objects are surprisingly similar to the flux tubes which form between two monopoles in Type-II superconductors and also the hypothesized color field flux tube that is thought to form between two quarks in the QCD vacuum.
\end{abstract}

*E-mail address: dzhun@freenet.bishkek.su

${ }^{\dagger}$ E-mail address:das3y@maxwell.phys.csufresno.edu 


\section{INTRODUCTION}

From Einstein and Wheeler down to the present day wormholes (WH) have been one of the most intriguing objects of study in general relativity. For example, if WHs exist in nature then what is the linear size of the mouth of the wormhole? If the mouth is sufficiently large then one has a classical gravitational object which can carry matter and energy between different spacetime points [1] ! If the mouth size is small (approximately the Planck length $\left.\approx L_{P l}\right)$ then one has a gravitational object which could play an important role in spacetime foam, and may even serve as a geometrical model for material particles such as the electron

[2], [3]. Wheeler coined the name "charge without charge" and "mass without mass" for such objects.

In 4D Euclidean gravity WH solutions were obtained in [4]. A full review of this problem can be found in [5]. In Ref. [6] a WH-like solution in multidimensional gravity without any kind of matter was given. Our point of view is that such vacuum WHs should be important for Wheeler's "charge without charge" and "mass without mass" objects [0]. The flux tube solutions discussed in this paper represent a limiting case of the vacuum WH-like solutions, where the wormhole mouths are separated by an infinite distance, and the cross section size of the WH-like solution is constant. In addition, the string-like character of the flux tube solutions, as well as their similarity to the flux tube structures in Type II superconductors and the QCD vacuum in the presence of quarks, make them interesting objects of study.

\section{5D FLUX TUBE SOLUTION}

In this article we first examine the standard vacuum 5D Kaluza-Klein theory. We take the form of the metric as

$$
d s^{2}=e^{2 \nu(r)} d t^{2}-r_{0}^{2} e^{2 \psi(r)-2 \nu(r)}(d \chi+\omega(r) d t-n \cos \theta d \varphi)^{2}-d r^{2}-a(r)\left(d \theta^{2}+\sin ^{2} \theta d \varphi^{2}\right)
$$

where $\chi$ is the $5^{\text {th }}$ extra coordinate; $r, \theta, \varphi$ are $3 D$ "spherical-polar" coordinates (the quotations marks indicate that these coordinates are not the same as the standard spherical-polar 
coordinates of flat space); $n$ is an integer; $r \in\left\{-R_{0},+R_{0}\right\}$. The form of the metric and the limit of $\mathrm{r}$ indicates that our metric is that for a WH $\left(R_{0}\right.$ may be equal to $\left.\infty\right)$. As in Ref. [8] the presence of the $n \cos \theta d \varphi$ term indicates the possible presence of a "magnetic" charge in the solution. We consider 5D Kaluza-Klein theory as gravity on the principal bundle with a U(1) structural group and with ordinary 4D Einstein spacetime as the base space 99. In this kind of theory no quantities depend on the $5^{\text {th }}$ coordinate because the $5^{\text {th }}$ dimension is a symmetric space (the gauge group $\mathrm{U}(1))$.

Using a REDUCE package for symbolic calculation the 5D Einstein equations for the metric given in Eq. (11) are

$$
\begin{aligned}
\nu^{\prime \prime}+\nu^{\prime} \psi^{\prime}+\frac{a^{\prime} \nu^{\prime}}{a}-\frac{1}{2} r_{0}^{2} \omega^{\prime 2} e^{2 \psi-4 \nu} & =0, \\
\omega^{\prime \prime}-4 \nu^{\prime} \omega^{\prime}+3 \omega^{\prime} \psi^{\prime}+\frac{a^{\prime} \omega^{\prime}}{a} & =0, \\
\frac{a^{\prime \prime}}{a}+\frac{a^{\prime} \psi^{\prime}}{a}-\frac{2}{a}+\frac{Q^{2}}{a^{2}} e^{2 \psi-2 \nu} & =0, \\
\psi^{\prime \prime}+\psi^{\prime 2}+\frac{a^{\prime} \psi^{\prime}}{a}-\frac{Q^{2}}{2 a^{2}} e^{2 \psi-2 \nu} & =0, \\
\nu^{\prime 2}-\nu^{\prime} \psi^{\prime}-\frac{a^{\prime} \psi^{\prime}}{a}+\frac{1}{a}-\frac{a^{\prime 2}}{4 a^{2}}-\frac{1}{4} r_{0}^{2} \omega^{\prime 2} e^{2 \psi-4 \nu}-\frac{Q^{2}}{4 a^{2}} e^{2 \psi-2 \nu} & =0
\end{aligned}
$$

here $Q=n r_{0}$. We will find that this represents the Kaluza-Klein "magnetic" charge. A particularly simple solution of these equations is

$$
\begin{array}{r}
q=Q, \\
a=\frac{q^{2}}{2}=\text { const }, \\
e^{\psi}=e^{\nu}=\cosh \left(\frac{r \sqrt{2}}{q}\right), \\
\omega=\frac{\sqrt{2}}{r_{0}} \sinh \left(\frac{r \sqrt{2}}{q}\right)
\end{array}
$$

In order to define the Kaluza-Klein "electrical" field of this solution we multiple Eq. (3) by $4 \pi$ and rewrite it in the following way:

$$
\left(\omega^{\prime} e^{3 \psi-4 \nu} 4 \pi a\right)^{\prime}=0 .
$$

This can be compared with the normal 4D Gauss's Law 


$$
\left(E_{4 D} S\right)^{\prime}=0
$$

where $E_{4 D}$ is the $4 \mathrm{D}$ electric field and $S=4 \pi r^{2}$ is the area of the 2 -sphere $S^{2}$. For the solution given in Eqs. (77 - 10), where $r^{2}$ is replaced by $a=$ const, we find that the surface area of the flux tube solution is $S_{\text {flux }}=4 \pi a$. Using this fact and comparing Eqs. (11) and (12) we can identify $\omega^{\prime} e^{3 \psi-4 \nu}$ as the Kaluza-Klein "electric" field:

$$
E_{K K}=\omega^{\prime} e^{3 \psi-4 \nu}=\frac{2}{r_{0} q}=\frac{q}{r_{0} a}=\text { const. }
$$

The Kaluza-Klein "magnetic" field can be derived as in Ref. [8]. The gauge field associated with the metric in Eq. (11) has a $\varphi$ component as $A_{\varphi}=-r_{0} n \cos \theta$. The Kaluza-Klein "magnetic" field is then found from $\mathbf{H}_{K K}=\nabla \times \mathbf{A}$, where the curl is taken using the metric of Eq. (1) and the solution of Eqs. (10 - 10). The resultant Kaluza-Klein "magnetic" field derived from this is

$$
\mathbf{H}_{K K}=\frac{1}{a \sin \theta}\left(\frac{\partial}{\partial \theta}\left(-r_{0} n \cos \theta\right)\right) \hat{\mathbf{r}}=\frac{r_{0} n}{a} \hat{\mathbf{r}}=\frac{Q}{a} \hat{\mathbf{r}} .
$$

Thus, this solution is a flux tube with constant Kaluza-Klein "electrical" and "magnetic" fields. The tube-like geometry of this solution comes from the fact that $a(r)=$ const rather than $a(r)=r^{2}$. The metric function $a(r)$ can be thought of as the cross-sectional size of the space at a particular $r$. For the present solution the cross-sectional size of the space remains constant as $r$ is varied and one has a tube-like space. The unit vector $\hat{\mathbf{r}}$ then points along the axis of the tube. Similar flux tube like solutions were investigated by Davidson and Gedalin [10]. The direction of both the "electric" and "magnetic" fields is along the $\hat{\mathbf{r}}$ direction (i.e. along the axis of the flux tube). The sources of these Kaluza-Klein fields (5D "electric" and "magnetic" charges) are located at $r= \pm \infty$. This permits us to view this solution as a 5D "electric" and "magnetic" dipole.

It is interesting to compare the 5D solution of Eqs. (7) - (10) with the following $4 \mathrm{D}$, nonasymptotically flat, electrogravity solution which was originally investigated by LeviCivita [11] and rediscovered in Ref. [12]: 


$$
\begin{aligned}
& d s^{2}=a^{2}\left(\cosh ^{2} \zeta d t^{2}-d \zeta^{2}-d \theta^{2}-\sin ^{2} \theta d \varphi^{2}\right), \\
& F_{01}=\rho^{1 / 2} \cos \alpha, \quad F_{23}=\rho^{1 / 2} \sin \alpha
\end{aligned}
$$

where

$$
G^{1 / 2} a \rho^{1 / 2}=1
$$

$\alpha$ is an arbitrary constant angle; $a$ and $\rho$ are constants defined by Eq. (17); $G$ is Newton's constant ( $c=1$, the speed of light); $F_{\mu \nu}$ is the electromagnetic field tensor. For $\cos \alpha=1$ $(\sin \alpha=1)$ one has a purely electric (purely magnetic) field. Since our 5D solution had $G_{55}=$ const the solutions of Eqs. (7) $-(10)$ and Eqs. (15) $(\sqrt{17})$ are similar. However, our

$5 \mathrm{D}$ solution had the condition that $\alpha=\pi / 4\left(E_{K K}=H_{K K}\right)$. This constraint indicates a difference between the 5D and 4D cases. Generally speaking 5D gravity is equivalent to $4 \mathrm{D}$ gravity + electromagnetic + scalar fields [13]. For our 5D solution we find that as a consequence of the condition $E_{K K}=H_{K K}$ the 5D gravitational field solutions are identical to the 4D gravitational plus electromagnetic field solutions.

In Ref. [14 the 4D solution of Eqs. (15)-(17) was used to construct a composite WH. At the center of this composite WH the solution of Eqs. (15)-(17) was matched to two Schwarzschild solutions. A similar construction was carried out in Ref. [7] with one distinction: the center of the composite WH in [7] was a vacuum solution of 5D gravity with $G_{5 t} \neq 0$ (this led to the appearance of the 5D "electric" field).

\section{7D FLUX TUBE SOLUTION}

Next we examine 7D Kaluza-Klein theory where the gauge group of the fibre is now $\mathrm{SU}(2)$. We take our metric to be of the form

$$
d s^{2}=e^{2 \nu(r)} d t^{2}-r_{0}^{2} \sum_{a=1}^{3}\left(\sigma^{a}-A_{\mu}^{a}(r) d x^{\mu}\right)^{2}-d r^{2}-a(r)\left(d \theta^{2}+\sin ^{2} \theta d \varphi^{2}\right)
$$

This is a simplified form of the metric used in [6]. The one-forms $\sigma^{a}$ are given by 


$$
\begin{aligned}
\sigma^{1} & =\frac{1}{2}(\sin \alpha d \beta-\sin \beta \cos \alpha d \gamma) \\
\sigma^{2} & =-\frac{1}{2}(\cos \alpha d \beta+\sin \beta \sin \alpha d \gamma) \\
\sigma^{3} & =\frac{1}{2}(d \alpha+\cos \beta d \gamma)
\end{aligned}
$$

where $0 \leq \beta \leq \pi, 0 \leq \gamma \leq 2 \pi, 0 \leq \alpha \leq 4 \pi$ are Euler angles for the $\mathrm{SU}(2)$ group. The potentials, $A_{\mu}^{a}$, are chosen to have the following monopole like form

$$
\begin{aligned}
& A_{\theta}^{a}=\frac{1}{2}(\sin \varphi ;-\cos \varphi ; 0) \\
& A_{\varphi}^{a}=\frac{\sin \theta}{2}(\cos \varphi \cos \theta ; \sin \varphi \cos \theta ;-\sin \theta) \\
& A_{t}^{a}=v(r)(\sin \theta \cos \varphi ; \sin \theta \sin \varphi ; \cos \theta)
\end{aligned}
$$

Substituting the metric ansatz of Eq. (18) into the 7D Einstein vacuum equations we find, again using a REDUCE package for symbolic manipulations, the following set of coupled equations

$$
\begin{aligned}
\nu^{\prime \prime}+\nu^{\prime 2}+\frac{a^{\prime} \nu^{\prime}}{a}-\frac{r_{0}^{2}}{2} e^{-2 \nu}{v^{\prime}}^{2} & =0 \\
\nu^{\prime \prime}+\nu^{\prime 2}+\frac{a^{\prime \prime}}{a}-\frac{a^{2}}{2 a^{2}}-\frac{r_{0}^{2}}{2} e^{-2 \nu} v^{2} & =0 \\
\frac{a^{\prime \prime}}{a}+\frac{a^{\prime} \nu^{\prime}}{a}-\frac{2}{a}+\frac{r_{0}^{2}}{4 a^{2}} & =0 \\
\frac{r_{0}^{2}}{6} e^{-2 \nu} v^{\prime 2}-\frac{2}{r_{0}^{2}}-\frac{r_{0}^{2}}{24 a^{2}} & =0 \\
v^{\prime \prime}-v^{\prime}\left(\nu^{\prime}-\frac{a^{\prime}}{a}\right) & =0
\end{aligned}
$$

Eq. (29) can be integrated once to give

$$
v^{\prime}=\frac{q}{r_{0} a} e^{\nu}
$$

where $q$ is an integration constant. Inserting this result back into Eqs. (25 - 29) one finds a solution which is almost identical to the 5D case given by Eqs. (8- 10)

$$
\begin{aligned}
a & =\frac{2 q^{2}}{7}=\frac{r_{0}^{2}}{8}=\text { const } \\
e^{\nu} & =\cosh \left(\frac{7 r}{2 \sqrt{2} q}\right) \\
v & =\frac{\sqrt{2}}{r_{0}} \sinh \left(\frac{7 r}{2 \sqrt{2} q}\right)
\end{aligned}
$$


By comparing Eq. (33) and Eq. (30) we find that the integration constant $q=\sqrt{7 a / 2}$. This constant $q$ is the electric "charge" of the present solution. This 7D solution also has color "electric" and "magnetic" fields similar to those of the 5D solution. To see this most directly one can apply the following gauge transformation to the potentials of Eqs. (22 - 24)

$$
A_{\mu}^{\prime}=S^{-1} A_{\mu} S-i\left(\partial_{\mu} S^{-1}\right) S
$$

where

$$
S=\left(\begin{array}{cc}
\cos \frac{\theta}{2} & -e^{-i \phi} \sin \frac{\theta}{2} \\
e^{i \phi} \sin \frac{\theta}{2} & \cos \frac{\theta}{2}
\end{array}\right)
$$

with this gauge transformation we find that the gauge potentials become

$$
\begin{aligned}
& A_{\theta}^{\prime a}=(0 ; 0 ; 0) \\
& A_{\varphi}^{\prime a}=(\cos \theta-1)(0 ; 0 ; 1) \\
& A_{t}^{\prime a}=v(r)(0 ; 0 ; 1)
\end{aligned}
$$

So that only the $\sigma^{3}$ direction in isospin space is non-zero. The calculation of the KaluzaKlein "magnetic" and "electric" fields is now the same as for the 5D case since the "Abelian" gauge transformation given by Eqs. (34) - (35) brings us to a gauge where the non-Abelian fields take on an Abelian form. Putting back the factor of $r_{0}$ from the metric of Eq. (18) in the gauge potentials we find that the Kaluza-Klein "magnetic" field for the 7D case becomes

$$
\mathbf{H}_{K K}=H_{K K}^{3}=\frac{1}{a \sin \theta}\left(\frac{\partial}{\partial \theta}\left(r_{0} A_{\varphi}^{3}\right)\right) \hat{\mathbf{r}}=\frac{-r_{0}}{a} \hat{\mathbf{r}}
$$

(Compared to the 5D case, we have from the outset specialized to $n=1$ for the $7 \mathrm{D}$ case). The Kaluza-Klein "electric" field is given by

$$
\mathbf{E}_{K K}=E_{K K}^{3}=\frac{1}{e^{\nu}}\left(\frac{\partial}{\partial r}\left(r_{0} A_{t}^{3}\right)\right) \hat{\mathbf{r}}=\frac{7}{2 r_{0} q} \hat{\mathbf{r}}=\frac{q}{r_{0} a} \hat{\mathbf{r}}
$$

Thus, just as in 5D Kaluza-Klein theory, 7D Kaluza-Klein theory yields an infinite length flux tube solution. The constant cross section size of this solution is set by the constant, a. Also, as in the 5D case, the length of this tube is filled with uniform "electric" and "magnetic" fields. The 7D "electric" and "magnetic" charge which produce these fields are taken to be located at $r= \pm \infty$. 


\section{DISCUSSION}

We would like to emphasize the important role that the "magnetic" charge plays in the formation of these flux tubes. If the "magnetic" charge is zero (i.e. if $n=0$ ) then we would have a wormhole-like solution located between two null surfaces as in [6] [9]. The addition of "magnetic" charges results in the wormhole-like solutions becoming the flux tube solutions presented here. This situation has similarities to the formation of flux tubes in Type II superconductors. When a magnetic monopole and anti-monopole are placed within a Type II superconductor a flux tube will form between them. Also, in the dual superconductor picture of confinement for QCD, color "electric" monopoles are necessary in order that a color field flux tube form between two color "electric" charges. One difference is that in these cases the flux tube is usually only filled with "magnetic" or "electric" fields. For the solutions presented here both "electric" and "magnetic" fields occur within the flux tube.

The asymptotic behaviour of this flux tube solution is interesting. At infinity the timetime part of the $5 \mathrm{D}$ metric approaches $G_{t t}=\exp (2 \nu) \stackrel{r \rightarrow \pm \infty}{\longrightarrow} \infty$. This situation is easy to understand: at infinity the area of the $S^{2}$ sphere is $4 \pi a$ rather than $4 \pi r^{2}$. This means that our solution is not an asymptotically flat wormhole. This indicates that this solution, while probably having limited use as a macroscopic object as in [1], may be of interest as a Planck scale object in connection with ideas on spacetime foam or as a geometrical model for material objects such as electrons or strings.

\section{ACKNOWLEDGEMENTS}

This work has been funded by the National Research Council under the Collaboration in Basic Science and Engineering Program. 


\section{REFERENCES}

[1] M.S. Morris and K.S. Thorne, Am. J. Phys., 56, 395 (1988).

[2] A.Einstein and N. Rosen, Phys. Rev., 48, 73 (1935).

[3] J.A. Wheeler, Phys. Rev., 97, 511 (1955).

[4] S.Giddings and A. Strominger, Nucl.Phys., B306, 890 (1988).

[5] M. Visser, Lorentzian Wormholes: from Einstein to Hawking, (AIP, New York, 1995).

[6] V.D.Dzhunushaliev, Izv. Vuzov, ser.Fizika, N6, 78(1993)(in Russian); Grav. and Cosmol., 3, 240 (1997).

[7] V.D.Dzhunushaliev, "Multidimensional geometrical model of the renormalized electrical charge with splitting off the extra coordinates", awarded Honorable Mention by Grav. Res. Found., 1998; Mod. Phys. Lett. A, 13, 2179 (1998).

[8] D.J. Gross and M.J. Perry, Nucl. Phys. B226, 29 (1983)

[9] V.D.Dzhunushaliev, Gen. Rel. Grav., 30, 583 (1998).

[10] A. Davidson and E. Gedalin, Phys. Lett. B339, 304 (1994)

[11] T. Levi-Civita, Rend. Atti Acad. Naz. Lincei, 26, 529 (1917).

[12] B. Bertotti, Phys. Rev., 116, 1331 (1959); I. Robinson, Bull. Akad. Pol., 7, 351 (1959).

[13] A. Salam, and J. Strathdee, Ann.Phys. (NY) 141, 316 (1982); R. Percacci, J.Math.Phys. 24, 807 (1983).

[14] E.I. Guendelman, Gen. Rel. Grav., 23, 1415 (1991). 